\title{
Body mass index and blood pressure in rural, low socioeconomic children
}

\author{
Leah E. Robinson ${ }^{*}$, Colleen M. Daly, Danielle D. Wadsworth
}

School of Kinesiology, Auburn University, Auburn, USA

*Corresponding Author: ler0004@auburn.edu

Received 21 June 2013; revised 21 July 2013; accepted 5 August 2013

Copyright (C) 2013 Leah E. Robinson et al. This is an open access article distributed under the Creative Commons Attribution License, which permits unrestricted use, distribution, and reproduction in any medium, provided the original work is properly cited.

\section{ABSTRACT}

Background: Young children who are overweight and obese may be at a greater risk of hypertension, and those from rural and low socioeconomic areas may also exhibit a prevalence of metabolic dysfunction and cardiovascular risk. This study evaluated the relationship of body mass index (BMI) and blood pressure in children from a rural, low socioeconomic community in Alabama. Methods: This cross-sectional study was comprised of young children (aged 5 - 9 years). BMI (normal, overweight, obese) was assessed using standardized procedures and classified based on the Centers for Disease Control Growth Charts. Blood pressure status (normal, pre-hypertension, hypertension; stage 1 and stage 2) was defined by percentiles of the 2nd and 3rd reading on a single occasion. A MANOVA was used to determine the relationship of BMI to mean blood pressure (i.e., systolic and diastolic). Results: Of the 134 participants (78\% non-Hispanic Black), 10 (7.5\%) were overweight and $25(18.7 \%)$ were obese. Pre-hypertension and hypertension were detected in $9(6.7 \%)$ and 20 (14.9\%) children, respectively. Results indicate that mean systolic and diastolic blood pressure significantly increased with $\mathrm{BMI}, P<0.001$ and $P=0.028$. Conclusion: Hypertension was strongly associated with an unhealthy BMI in these young children from a rural and low-socioeconomic community. The findings raise concerns about the growing epidemic of childhood obesity and hypertension in young children and warrant the need to investigate lifestyle behavioral interventions on BMI and blood pressure.

Keywords: Hypertension; Rural Health; Pediatrics; Obesity; Overweight

\section{INTRODUCTION}

Over the past 30 years, the prevalence of overweight and obese children in the United States has more than tripled [1]. Currently, 1 out of 3 children in the United States between the ages of 2 - 19 years are overweight or obese and in the state of Alabama, 36\% of children are overweight or obese [1].

This increase in weight parallels an increase in chronic conditions and other health-related complications such as metabolic dysfunction, type-2 diabetes, asthma, sleep apnea, and gall-bladder diseases [2,3]. Although, hypertension, a condition in which the pressure in the arteries is continually elevated, is considered to be a rare occurrence in young children; studies have shown a rise in this condition in children over the past two decades [4-6]. Early identification is important because hypertension during the childhood years is a predictive indicator for hypertension in the adult years [7-9]. Hypertension is also a risk factor of coronary artery disease in adults and could be a warning sign for the early development of this disease along with atherosclerosis in young children [10].

Recent studies, both national and international, have identified an association between BMI and hypertension in children between the ages of $1-17$ years [11-15]. Excess body weight has been shown to increase systolic blood pressure (SBP) by $1.4 \mathrm{mmHg}$ and $3.3 \mathrm{mmHg}$ in diastolic blood pressure (DBP) $[16,17]$. With the increasing diagnosis of hypertension in children and youth, there is a need for early detection of hypertension in order to better inform intervention and educational programs that aid in preventing long-term complications of hypertension.

National studies provide a depiction of the health trends; however, as it relates to young rural, poor children in Alabama this picture may be even more problematic and warrant additional inquiry and possibility action. Few studies have examined the effect of weight status 
and its effect on blood pressure in a rural population from the Southern region of the United States. Therefore, the aim of this study was to evaluate the relationship between BMI and blood pressure in young children living in a rural, low socioeconomic community in Alabama. This study also examined the prevalence of overweight and obese children in a rural community.

\section{METHODS}

The data for this cross-sectional study was collected in a rural elementary school in Alabama. The primary school comprised of grades K-6 and 316 students (52\% females, $48 \%$ males) at the time of data collection. Black children (85\%) were the predominant racial/ethnic group that attended the school. The median household income in the school was $\$ 30,000$ USD made up of less than 1,000 residents who are majority of Black ancestry. For the study, K-2nd graders enrolled in the school served as the participants for this study. A recruitment letter and consent/assent form was distributed to the parent of each child. Parental consent and child assent were obtained in accordance with the project protocol approved by the Institutional Review Board at Auburn University. At the time of data collection, none of the participants were taking antihypertensive medication or had previously received a diagnosis of hypertension. Participant demographics are located in Table 1.

Data (height, weight, and resting blood pressure) were collected in the schools from September 2010 to October 2010 by trained research assistants. Racial and ethnic classification and date of birth information was collected from school records.

Body Mass Index. Height was measured to the nearest 0.1 centimeter using Digital Medical Scales (Seca Floor Scale 769, SECA Corp. Hanover, MD). The measurement of height was conducted with shoes, coats, and other heavy outerwear removed. The children were instructed to keep their shoulders in the relaxed position, their arms hanging freely and their head aligned in the Frankfurt plane. This scale was also used to record body weight, which we measured to the nearest $0.1 \mathrm{~kg}$. BMI was calculated as: [weight $(\mathrm{kg}) /$ height $(\mathrm{m})^{2}$ ]. BMI percentiles were classified according to the CDC classification's age- and sex-specific BMI cutoff points for "normal weight” (84th percentile and below), "overweight" (85th to 94th percentile), and "obese" (95th and above).

Resting Blood Pressure. Resting blood pressure was measured with the Omron HEM-711 DLX Automatic Blood Pressure Monitor (Omron Healthcare, Inc, Vernon Hill, IL) using a cuff size that was appropriate for the measured arm circumference in accordance with the National High Blood Pressure Education Program Working Group. Three blood pressure readings were taken in a seated position with arm resting in a palm facing upward.
Table 1. Participants demographic.

\begin{tabular}{cc}
\hline Total & 134 participants \\
\hline Age in years (mean \pm SD) & 6.45 (1.02) years \\
Grade & 40 Kindergarteners \\
& 53 1st graders \\
Sex & 21 2nd graders \\
& 69 Boys \\
Race/Ethnicity & 65 Girls \\
& $78 \%$ non-Hispanic Black \\
BMI (mean \pm SD) & $17 \%$ Hispanic \\
Range & $5 \%$ non-Hispanic White \\
Range & 16.82 (3.682) \\
Systolic Blood Pressure (mean \pm SD) & 102.54 (9.701) mm Hg \\
Riastolic Blood Pressure (mean \pm SD) & 80 - 129 \\
Range & 45 - 86 \\
\hline
\end{tabular}

Readings were collected from each child following a 3-minute relaxing activity (i.e., coloring activity). Between each of the three blood pressure measurements, a 1-minute rest period occurred and the child returned to the coloring activity. The mean of the last two SBP and DBP was used for the classification of the children's hypertension status. BP was reassessed the following week for children with a difference of $10 \mathrm{mmHg}$ between the last two BP readings and/or a SBP greater than 120 $\mathrm{mmHg}$. Blood pressure was classified according to the 2004 National High Blood Pressure Education Program Working Group on High Blood Pressure in Children and Adolescents. The classifications are defined in accordance with the child's height, age, and sex. The definitions of pre-hypertension and hypertension were as follows: "normal": SBP and DBP <90th percentile, "Pre-hypertension": SBP or DBP 90th percentile to $<95$ th percentile, and "Hypertension" $\geq 95$ th percentile.

\section{Analysis}

All statistical analyses were analyzed with the PSAW Statistics 18 (IBM Corporation; Somers, NY). Descriptive statistics was used to describe BMI, SBP, and DBP. A multivariate analysis of variance (MANOVA) was used to determine the relationship of BMI category (normal, overweight, obese) on mean SBP and DBP. Post hoc analyses were assessed with Bonferroni adjustment. All significance tests were based at $p=0.05$.

\section{RESULTS}

A total of 134 children with a mean age $6.45 \pm 1.02$ years served as participants in this study. The sample was comprised of 65 girls and 69 boys and a majority of the participants were non-Hispanic Black (78\%). Table 1 
provides an overview of the demographical characteristics. The number of children who were overweight or obese was 10 (7.5\%) and 25 (18.7\%), respectively. Prehypertension and hypertension was detected in 29 children. Nine $(6.7 \%)$ children were considered to be prehypertensive, while 20 (14.9\%) were classified as hypertensive. Characteristics of the study across BP and weight status are listed in Table 2. Findings indicate that there was no effect of sex, nor an interaction of sex and BMI status on SBP and DBP, $P>0.05$. However, results did indicate that mean SBP and DBP were significantly affected by an increased BMI, $F_{(2,128)}=16.54, P<0.001$ and $F_{(2,128)}=3.69, P=0.028$. Post hoc analyses confirmed that overweight and obese children exhibited markedly higher SBP than normal weight children. Only obese children demonstrated significantly higher DBP compared to normal and overweight children.

\section{DISCUSSION}

This study examined the effect of BMI status on blood pressure in young children living in a low socioeconomic, rural setting. Results from this study show that hypertension is present in young rural children and that children with an unhealthy weight status demonstrate unhealthy blood pressure status compared to those who fall within the recommended BMI weight classification [18,19]. Our findings are consistent with several studies that have shown a strong association between elevated BMI and high values of BP in youth [12-19]. Much of the research examining blood pressure and obesity relationships in children have been conducted by The Bogalusa Heart Study, which is a long-term community-based epidemicologic study of the early natural history of cardiovascular disease in children and young adults. The Bogalusa Heart Study participants are from a semirural, biracial (65\% white, 35\% black) area in Louisiana (The Bogalusa Heart Study 20th anniversary). Multiple studies based on the Bolgalusa Heart Study have shown that obesity is an important determinant of high blood pressure levels [20], and this association begins during the childhood years [21]. This relationship also holds true for this study where residences are predominately black (86\%) and reside in a small (population less than 1,000 ) rural, poor area in Southeast Alabama.

An interesting finding that evolved from this study that has not been reported in others is the association between obesity and DBP. Specifically, only obese children demonstrated significantly higher DBP. This study is limited and definitive conclusions could not be made as it relates to why only obese children were classified with an elevated DBP status. However, this study does show the effect that additional weight may have on the heart, specifically when in the resting phase of the cardiac cycle.
Table 2. Characteristics of SBP and DBP based on BMI classification.

\begin{tabular}{cccc}
\hline $\begin{array}{c}\text { Categories } \\
\text { based on BMI }\end{array}$ & $\begin{array}{c}\text { Normal } \\
\boldsymbol{n}(\mathbf{\%})\end{array}$ & $\begin{array}{c}\text { Pre-Hypertension } \\
\boldsymbol{n}(\mathbf{\%})\end{array}$ & $\begin{array}{c}\text { Hypertension } \\
\boldsymbol{n}(\mathbf{\%})\end{array}$ \\
\hline Normal & & & \\
Boys SBP & $41(83.6)$ & $4(8.2)$ & $4(8.2)$ \\
Girls SBP & $45(90.0)$ & $3(6.0)$ & $2(4.0)$ \\
Boys DBP & $48(98.8)$ & $1(1.2)$ & $0(0.0)$ \\
Girls DBP & $50(100.0)$ & $0(0.0)$ & $0(0.0)$ \\
Overweight & & & \\
Boys SBP & $4(66.7)$ & $0(0.0)$ & $2(33.3)$ \\
Girls SBP & $2(50.0)$ & $0(0.0)$ & $2(50.0)$ \\
Boys DBP & $6(100.0)$ & $0(0.0)$ & $0(0.0)$ \\
Girls DBP & $3(75.0)$ & $1(25.0)$ & $0(0.0)$ \\
Obese & & & \\
Boys SBP & $7(50.0)$ & $1(7.1)$ & $6(42.9)$ \\
Girls SBP & $6(54.5)$ & $1(2.6)$ & $4(42.9)$ \\
Boys DBP & $0(0.0)$ & $0(0.0)$ & $14(100.0)$ \\
Girls DBP & $0(0.0)$ & $1(9.1)$ & $10(90.9)$ \\
\hline
\end{tabular}

Blood pressure increases with age and race plays a role in this increase. Specifically, Black children have a higher prevalence of elevated BP (95th percentile) at lower levels of BMI, but among children with the highest levels of BMI, more White children have elevated BP than Black children [22]. In addition, Black children with primary hypertension around age 13 may be at increased cardiovascular risk compared with non-Black children with primary hypertension [22]. Although this sample is too small to examine racial differences, a high percentage (78\%) of Black participants may factor into the relationship between BP and DBP. Of a further note, most studies examining the studies relationship between BMI, $\mathrm{BP}$ and race are older ( $>$ than 10 years of age) compared to the sample from this study (mean age $6.45 \pm 1.02$ ). To obtain a better understanding of this, future studies should incorporate various measures to assess vascular function (i.e., computerized and ultrasonic procedures). Additionally, it would be beneficial for studies to examine flow mediated dilation and/or arterial stiffness along with resting blood pressure to get a more thorough understanding of the relationship of BMI and resting blood pressure in children. It should be noted that systolic hypertension is more common than diastolic hypertension, but high DBP could be an indication of diastolic heart failure $[23,24]$. Thus, future studies should examine DBP more critically in young children as a means for early detection of cardiovascular risk.

The finding that BMI is positively associated with an 
increase in SBP and DBP is important to the long-term health of young children since both variables track from childhood to adulthood [7-9,25] and the prevention of hypertension is linked with body weight early in life [26]. This is a worthy issue from a public and school health standpoint since the prevalence of hypertension in childhood and adolescence appears to be strongly associated with higher morbidity and mortality related to cardiovascular disease during the adult years [16].

We acknowledge that there were limitations to this current study. First, this study included a relatively small sample size and the population was young children. From the literature, we are confident that the findings will hold true in a larger sample size and the propensity of ages. Specifically, National Health and Nutrition Examination Survey for children between the ages of 8 - 17 years indicated that SBP and DBP have increased over the past decade and the increase was partially attributed to an unhealthy increase in BMI [6]. Other limitations include the absence of assessing family medical history. This information could provide clues as it relates to any hereditary medical conditions. Specifically, it could identify if a child may have a higher-than-usual chance for such conditions like heart disease, hypertension, and diabetes. These complex conditions might be due to a combination of genetics, environmental factors, and lifestyle choices. Therefore, future studies should include additional variables that influence weight status and hypertension such as nutritional intake and physical activity participation, and how school and community policies contribute to or reduce health behaviors that would affect hypertension. Additionally, the measurement of BMI has limitations because it is possible for a child to be overweight but not fat. Therefore, it is possible for participant misclassification when weight status is based on BMI alone because it does not take into account lean muscle mass. However, the correlation among BMI and body fat are extremely high [27].

\section{CONCLUSION}

Findings from this study support that children are demonstrating an early warning sign for potential health problems [9]. Hypertension was strongly associated with an unhealthy BMI. Specifically, nearly 1 out of every 3 children who participated in the study was either overweight or obese or had a blood pressure reading of prehypertensive or Stage 1 hypertension. This is a public health concern. There is a need for early screening programs to identify children who are at-risk along with behavioral (lifestyle) intervention programs that are designed to eliminate or reduce hypertension and other cardiovascular risk factors. The programs need to focus on promoting physical activity and healthy eating while integrating the family unit.

\section{ACKNOWLEDGEMENTS}

The authors would like to thank the public school (i.e., administrators, teachers, and students) for all of their participation and patience.

\section{REFERENCES}

[1] Ogden, C.L., Carroll, M.D., Curtin, L.R., et al. (2010) Prevalence of high body mass index in US children and adolescents, 2007-2008. JAMA, 303, 242-249. doi:10.1001/jama.2009.2012

[2] Daniels, S.R. (2006) The consequences of childhood overweight and obesity. Future of Children, 16, 47-67. doi:10.1353/foc.2006.0004

[3] Ebbeling, C.B., Pawlak, D.B. and Ludwig, D.S. (2002) Childhood obesity: Public-health crisis, common sense cure. Lancet, 360, 473-482. doi:10.1016/S0140-6736(02)09678-2

[4] Raitkari, O.T., Porkka, K.V.K., Rasanen, L., et al. (1994) Clustering and six year cluster-tracking of serum total cholesterol, HDL-cholesterol and diastolic blood pressure \&in children and young adults-the cardiovascular risk in young finns study. Journal of Clinical Epidemiology, 47, 1085-1093. doi:10.1016/0895-4356(94)90094-9

[5] Bao, W., Threefoot, S.A., Srinvasan, S.R., et al. (1995) Essential hypertension predicted by tracking of elevated blood pressure from childhood to adulthood: The Bogalusa heart study. American Journal of Hypertension, 8, 657-665. doi:10.1016/0895-7061(95)00116-7

[6] Muntner, P., He, J., Cutler, J.A., et al. (2004) Trends in blood pressure among children and adolescents. Pediatrics, 291, 2107-2113.

[7] Guo, S., Salisbury, S., Roche, A.F., et al. (1994) Cardiovascular disease risk factors and body composition: A review. Nutrition Research, 14, 1721-1777. doi:10.1016/S0271-5317(05)80327-6

[8] Gerber, L.M. and Stern, P.M. (1999) Relationship of body size and body mass to blood pressure: Sex-specific and developmental influences. The Program in Human Biology, 71, 505-528.

[9] Eisenmann, J.C. (2004) Physical activity and cardiovascular disease risk factors in children and adolescents: An overview. Canadian Journal of Cardiology, 20, 195-301.

[10] Luma, G.B. and Spiotta, R.T. (2006) Hypertension in children and adolescents. American Family Physician, 73, 1558-1568.

[11] Hanevold, C., Waller, J., Daniels, S., et al. and International Pediatric Hypertension Association. (2004) The effects of obesity, gender, and ethnic group on left ventricular hypertrophy and geometry in hypertensive children: A collaborative study of the International Pediatric Hypertension Association. Pediatrics, 113, 328-333. doi:10.1542/peds.113.2.328

[12] Andelopoulos, P.D., Milionis, H.J., Maochonis, G., et al. (2006) Relations between obesity and hypertension: Preliminary data from a cross-sectional study in primary 
schoolchildren: The children study. European Journal of Clinical Nutrition, 60, 1226-1234. doi:10.1038/sj.ejcn.1602442

[13] Antaei, N., Hosseini, M. and Iranmanesh, M. (2009) The relationship of body mass index and blood pressure in Iranian children $<7$ years old. Journal of Tropical Pediatrics, 55, 313-317. doi:10.1093/tropej/fmp014

[14] Moore, W.E., Stephens, A., Wilson, T., et al. (2006) Body mass index and blood pressure screening in a rural public school system: The Healthy kids project. Preventing Chronic Disease, 3, 1-10.

[15] Savadori, M., Sontrop, J.M., Garg, A.X., et al. (2008) Elevated blood pressure in relation to overweight and obesity among children in a rural Canadian community. Pediatrics, 122, e821-e827. doi:10.1542/peds.2008-0951

[16] Falkner, B., Gidding, S.S., Ramirez-Garnica, G., et al. (2006) The relationship of body mass index and blood pressure in primary care pediatric patients. Journal of Pediatrics, 128, 195-200. doi:10.1016/j.jpeds.2005.10.030

[17] Hlaing, W.M., Prineas, R.J. and Zhu, Y. (2006) Tragectory of systolic blood pressure in children and adolescents. Annals of Epidemiology, 16, 11-18. doi:10.1016/j.annepidem.2005.03.006

[18] Sorof, J.M., Lai, D., Turner, J., et al. (2004) Overweight, ethnicity and the prevalence of hypertension in schoolaged children. Pediatrics, 113, 475-482. doi:10.1542/peds.113.3.475

[19] Thompson, D.R., Obarzanek, E., Franko, D.L., et al. (2007) Childhood overweight and cardiovascular disease risk factors: The national heart, lung, and blood institute growth and health study. Journal of Pediatrics, 150, 18-25. doi:10.1016/j.jpeds.2006.09.039

[20] Brown, C.D., Higgins, M., Donato, K.A., et al. (2008) Body mass index and the prevalence of hypertension and dyslipidemia. Obesity Research, 8, 605-619. doi:10.1038/oby.2000.79

[21] Freedman, D.S., Dietz, W.H., Srinivasan, S.R., et al. (1999) The relation of overweight to cardiovascular risk factors among children and adolescents: The Bogalusa heart study. Pediactrics, 103, 1175-1182. doi:10.1542/peds.103.6.1175

[22] Rosner, B., Prineas, R., Daniels, S.R., et al. (2000) Blood pressure differences between blacks and whites in relation to body size among US children and adolescents. American Journal of Epidemiology, 151, 1007-1019. doi:10.1093/oxfordjournals.aje.a010129

[23] Kannel, W.B. (2000) Elevated systolic blood pressure as a cardiovascular risk factor. American Journal of Cardiology, 85, 251-255. doi:10.1016/S0002-9149(99)00635-9

[24] Chobanian, A.V., Karris, G.L., Black, H.R., et al. (2003) Evaluation, and treatment of high blood pressure seventh report of the joint national committee on prevention detection. Hypertension, 42, 1206-1252. doi:10.1161/01.HYP.0000107251.49515.c2

[25] Forsen, T., Nissinen, A., Tuomilehto, J., et al. (1999) Growth in childhood blood pressure in Finnish children. Journal of Human Hypertension, 12, 397-402. doi:10.1038/sj.jhh.1000643

[26] Eisenmann, J.C., Wrede, J. and Heelan, K.A. (2005) Association between adiposity, family history of CHD and blood pressure in 3-8 year-old children. Journal of $\mathrm{Hu}$ man Hypertension, 19, 675-681. doi:10.1038/sj.jhh.1001882

[27] Field, A.E., Laird, N., Steinberg, E., et al. (2003) Which metric of relative weight best captures body fatness in children? Obesity Research, 11, 1345-1352. doi:10.1038/oby.2003.182 